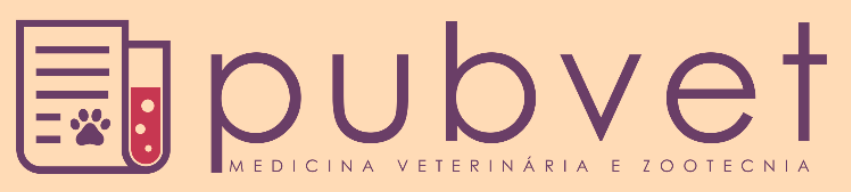

https://doi.org/10.31533/pubvet.v13n12a472.1-8

\title{
Características reprodutivas de cutias fêmeas (Dasyprocta prymnolopha wagler, 1831) criadas em cativeiro no nordeste do Brasil
}

\author{
João Augusto Rodrigues Alves Diniz ${ }^{1} \bullet$, Lilianne Marinho dos Santos Azerêdo ${ }^{3} \bullet$, Ediane Freitas \\ Rocha $^{2}{ }^{\circ}$, Artur da Nóbrega Carreiro ${ }^{\circ}$, Brunna Muniz Rodrigues Falcão ${ }^{2}{ }^{\circ}$, Joyce Galvão de \\ Souza $^{1 \bullet}$, Maria Acelina Martins de Carvalho ${ }^{\circ}{ }^{\circ}$, Danilo José Ayres de Menezes ${ }^{4 *}$ \\ ${ }^{I}$ Aluno (a) de Mestrando no Programa de Pós-Graduação em Ciência e Saúde Animal, Universidade Federal de Campina Grande, Patos, Paraíba, Brasil. \\ ${ }^{2}$ Aluna (o) de Doutorando no Programa de Pós-Graduação em Ciência e Saúde Animal, Universidade Federal de Campina Grande, Patos, Paraíba, Brasil. \\ ${ }^{3}$ Mestre em Medicina Veterinária pelo Programa de Pós-Graduação em Medicina Veterinária, Universidade Federal de Campina Grande, \\ Patos, Paraíba, Brasil. \\ ${ }^{4}$ Professora do Departamento de Morfofisiologia Veterinária, Universidade Federal do Piauí, Centro de Ciências Agrárias, Teresina, Piauí, Brasil \\ ${ }^{5}$ Professor do Departamento de Ciências Biológicas, Universidade Federal do Rio Grande do Norte, Centro de Biociências, Natal, Rio Grande \\ do Norte, Brasil. \\ *Autor para correspondência: mdanayres@gmail.com
}

Resumo. Objetivou-se com este trabalho realizar o levantamento de dados reprodutivos de cutias fêmeas criadas em cativeiro, durante o período de 1998 a 2007 e associá-los aos fatores climáticos da região, verificando a presença ou ausência de estacionalidade na reprodução. Foram avaliadas 178 fichas de acompanhamento individual de cutias nascidas no Núcleo de Estudos e Preservação de Animais Silvestres - NEPAS da UFPI em Teresina-PI. Por meio das fichas, foram anotadas as datas dos partos, o tipo de parto e a quantidade de filhotes por parto de cada fêmea. Os dados climáticos da região foram cedidos pela Empresa Brasileira de Pesquisa Agropecuária - EMBRAPA Meio Norte -Teresina, Piauí. Foi verificado um total de 129 partos durante esse período. Os resultados mostram uma maior concentração de partos entre os meses de julho a fevereiro, outubro com mais nascimentos, e diminuição entre março e junho. Estes resultados podem apontam para um aumento de atividade reprodutiva que coincide com os períodos mais quentes do ano e com menor volume de chuvas, havendo correlação positiva entre a temperatura máxima e média, e negativa com a umidade relativa do ar em relação ao aumento dos índices de nascimento. A atividade reprodutiva da cutia, assim como em muitos animais, pode está associada a inúmeros fatores, porém, há uma forte correlação às condições ambientais, indicando que os nascimentos se concentram em períodos do ano que precede ao de maior oferta de alimento, onde os animais estarão perdendo a dependência da mãe no período propício a sobrevivência.

Palavras-chave: estacionalidade, fatores climáticos, reprodução

\section{Reproductive characteristics of female agouti (Dasyprocta prymnolopha) created in captivity in northeast Brazil}

\begin{abstract}
The objective of this work was to survey the reproductive data of four females reared in captivity during the period 1998 to 2007 and to associate them with the climatic factors of the region, verifying the presence or absence of seasonality in reproduction. A total of 178 individual monitoring kits were evaluated. These were born at the Center for the Study and Preservation of Wild Animals (NEPAS) of the UFPI in Teresina-PI. By means of the records, the dates of the births, the type of delivery and the number of pups per delivery of each female were recorded. The climatic data of the region were provided by the Brazilian Agricultural Research Company - EMBRAPA Meio Norte -Teresina, Piauí. A total of 129 deliveries were recorded during this period. The results show a higher
\end{abstract}


concentration of births between the months of July to February, October with more births, and decrease between March and June. These results may point to an increase in reproductive activity that coincides with the hottest periods of the year and with a lower volume of rainfall. There is a positive correlation between maximum, average and negative temperature with relative humidity, of birth. However, there is a strong correlation with environmental conditions, indicating that births are concentrated in periods of the year preceding the one with the highest food supply, where animals will lose their dependence on the mother during the period of survival.

Keywords: seasonality, climatic factors, reproduction

\title{
Características reproductivas de Agouti hembra (Dasyprocta prymnolopha wagler, 1831) criada en cautiverio en el noreste de Brasil
}

\begin{abstract}
Resumen. El objetivo de este estudio fue recopilar los datos reproductivos de agutíes hembras criadas en cautiverio durante el período de 1998 a 2007 y asociarlos con los factores climáticos de la región, verificando la presencia o ausencia de estacionalidad en la reproducción. Se evaluaron un total de 178 registros de seguimientos individuales de agutíes nacidos en el Centro de Estudios y Conservación de Animales Salvajes - NEPAS de UFPI en Teresina-PI. A través de los registros, se registraron las fechas de los nacimientos, el tipo de parto y el número de crías por nacimiento de cada hembra. Los datos climáticos de la región fueron proporcionados por la Corporación Brasileña de Investigación Agrícola - EMBRAPA Middle North-Teresina, Piauí. Se verificaron un total de 129 partos durante este período. Los resultados muestran una mayor concentración de nacimientos entre julio y febrero, octubre con más nacimientos, y una disminución entre marzo y junio. Estos resultados pueden apuntar a un aumento en la actividad reproductiva que coincide con los períodos más calurosos del año y una menor precipitación, con una correlación positiva entre la temperatura máxima, media y negativa con la humedad relativa en relación con el aumento de los índices de nacimiento. La actividad reproductiva del agutí, como en muchos animales, puede estar asociada con muchos factores, sin embargo, existe una fuerte correlación con las condiciones ambientales, lo que indica que los nacimientos se concentran en períodos del año anterior al mayor suministro de alimentos, donde los animales perderán la dependencia de su madre en el período favorable para la supervivencia.
\end{abstract}

Palabras clave: estacionalidad, factores climáticos, reproducción

\section{Introdução}

Os estudos relativos à biologia de animais silvestres e o incremento dos conhecimentos sobre algumas espécies, particularmente relativo à reprodução, vem colaborando com o crescimento da produção animal, onde algumas espécies podem mostrar melhor adaptação em cativeiro e bom desempenho zootécnico. Neste contexto, inclui-se a cutia, um roedor silvestre, com um grande potencial para exploração econômica (Hosken \& Silveira, 2001; Pinheiro et al., 1989)

De acordo com Almeida et al. (2003), o conhecimento de aspectos biológicos e reprodutivos básicos de um animal e sua relação com outras espécies, além de melhorar o seu manejo produtivo e a sua exploração econômica, torna possível sua utilização como modelo experimental pelos pesquisadores.

A cutia (Dasyprocta prymnolopha) é uma espécie pertencente à ordem Rodentia e da família Dasyproctidae. Caracterizada por ser um roedor de médio porte, chegando a uma média de peso de aproximadamente $4 \mathrm{~kg}$, possui uma distribuição ampla, sendo encontrada em boa parte do território brasileiro (Hosken \& Silveira, 2001; Lange et al., 2014; Lange \& Schmidt, 2014). Dispõe de uma coloração avermelhada, com membros pélvicos longos e membros torácicos curtos, que auxiliam a leva o alimento a boca na hora da alimentação (Rêgo et al., 2019), e com unhas bastante forte, devido a seu hábito de escavar. Seus pares de dentes incisivos são longos estendendo-se para fora da boca, característica essa que facilita a sua alimentação na hora de abrir alimentos mais sólidos (Hosken \& Silveira, 2001). 
As investigações sobre os aspectos reprodutivos da cutia merecem destaque e, embora enriqueçam o acervo do conhecimento humano, ainda são insuficientes para garantir a adequada reprodução dessa espécie, seja em cativeiro ou mesmo em vida livre (Rodrigues et al., 2003). Dessa forma, observações do comportamento reprodutivo da cutia tornam-se de suma importância para a busca de biotecnologias reprodutivas que venham a melhorar sua reprodução em cativeiro, permitindo a interferência do homem neste aspecto, possibilitando traçar melhores técnicas de manejo em épocas do ano com melhor índice reprodutivo, ou mesmo, fornecer melhores condições em épocas de menor atividade reprodutiva que venham a estimular a reprodução desses animais nestes períodos.

Este trabalho teve como objetivo verificar o padrão de partos durante o ano de cutias criadas em cativeiro, realizando o levantamento dos dados reprodutivos desses animais entre 1998 e 2007 (período de 10 anos), assim como associar os dados reprodutivos levantados ao período do ano e determinar associações dos dados reprodutivos das cutias a fatores climáticos da região.

\section{Material e métodos}

Foram avaliadas 178 fichas de acompanhamento individual de cutias nascidas no Núcleo de Estudos e Preservação de Animais Silvestres - NEPAS (Registro IBAMA N ${ }^{\circ}$ 02/08-618), da Universidade Federal do Piauí, Teresina, Piauí, Brasil durante os anos de 1998 a 2007, período em que o NEPAS disponibilizou as fichas. O NEPAS fica localizado na cidade de Teresina $\left(05^{\circ} 05^{\prime} \mathrm{S}\right.$ e $42^{\circ} 48^{\prime} \mathrm{W}, 72 \mathrm{~m}$ a.s.l.), que possui clima predominante classificado como tropical subsumido quente. A região apresenta uma curta estação chuvosa com média de cinco meses, de janeiro a maio, com duração do fotoperíodo que oscila entre os horários de 12:03 a 12:37, durante todo o ano (Köppen \& Geiger, 1928).

Os animais viviam no NEPAS em grupos familiares, em baias de 3 x $9 \mathrm{~m}$ e com média de 24 espécimes por baia. Eram alimentadas com ração comercial peletizada (composta por: proteína bruta mínima-12\%, extrato etéreo- $1,5 \%$, matéria fibrosa- $12 \%$, matéria mineral-13\%, cálcio-1,3\% e fósforo0,4\%), além de milho, frutas e verduras regionais da época, e água ad libitum.

Por meio das fichas, foram anotadas as datas dos partos, o intervalo entre parto, o tipo de parto, simples, duplo ou triplo e a quantidade de filhotes por parto de cada fêmea. Com a obtenção desses dados, foi verificada a presença de partos nos meses do ano e se havia predominância em determinada época do ano.

Os dados climáticos da região foram solicitados à Empresa Brasileira de Pesquisa Agropecuária EMBRAPA Meio Norte -Teresina, Piauí. Os dados obtidos sobre o clima foram associados aos dados referentes ao nascimento dos animais fazendo-se a correlação entre o período de nascimento dos animais e as condições climáticas do período do ano de 1998 a 2007, por meio do programa ASSISTAT Versão 7.7 betas (Silva, 2016).

Nenhum animal foi manuseado neste estudo, dispensando, dessa forma, a aprovação de comitê de ética, conforme informado pelo CEUA/UFCG.

\section{Resultados e discussão}

Durante o levantamento das fichas foram contabilizados 149 partos, destes, 129 partos tinham a identificação da mãe e foram utilizados para verificar a ocorrência de um período de estacionalidade em cutias fêmeas criadas em cativeiro na cidade de Teresina, Piauí, Brasil.

Durante o período de 1998 a 2007, período em que o NEPAS manteve seus registros atualizados, os partos tiveram sua maior concentração nos meses de julho a fevereiro, decrescendo nos meses de março a junho, com nenhum ou quase nenhum nascimento nestes meses (Tabela 1).

O período de maior número de animais nascidos ocorreu concomitante ao período de menor umidade relativa do ar e maior temperatura (Tabelas 2 e 3), correspondendo ao período seco da região. Estes resultados apontam para uma estacionalidade reprodutiva, corroborando com Ptaszynska (2008), que afirma que a maioria dos animais silvestres e alguns animais domesticados apresentam como características comum a estacionalidade reprodutiva, possibilitando um maior número de nascimentos em um determinado momento do ano, proporcionando assim, aos recém-nascidos crescer sob ótimas condições de clima e disponibilidade de alimento. 
De acordo com Weir (1971), as cutias da espécie Dasyprocta agouti, criadas em cativeiro, apresentam um período de sazonalidade reprodutiva durante o verão Londrino, período que corresponde a temperaturas mais elevadas e dias mais longos, o que pode apontar semelhança ao comportamento dos animais no Nordeste do Brasil, porém, não havendo influência de fotoperíodo nestes últimos. Por outro lado, os dados levantados no NEPAS divergem de estudos realizados nas espécies D. aguti (Cavalcante et al., 2006), D. prymnolopha (Guimarães, 1993), D. punctata (Meritt Junior, 1983) e para outras espécies da família Dasyproctidae (Roth-Kolar, 1957; Zuckerman, 1952) em que afirmam que os nascimentos ocorrem durante todos os meses do ano.

Tabela 1. Quantidade de cutias (Dasyprocta prymnolopha) nascidas no NEPAS na UFPI-Teresina, no período de 1998 a 2007

\begin{tabular}{lccccccccccccc}
\hline & Jan & Fev & Mar & Abr & Mai & Jun & Jul & Ago & Set & Out & Nov & Dez & Total \\
\hline 1998 & 0 & 0 & 0 & 0 & 1 & 0 & 0 & 0 & 0 & 0 & 1 & 0 & 2 \\
1999 & 0 & 0 & 0 & 0 & 0 & 0 & 1 & 0 & 1 & 1 & 0 & 0 & 3 \\
2000 & 1 & 0 & 0 & 0 & 0 & 0 & 0 & 0 & 0 & 3 & 0 & 1 & 5 \\
2001 & 1 & 0 & 1 & 1 & 0 & 0 & 0 & 1 & 0 & 2 & 0 & 2 & 8 \\
2002 & 2 & 0 & 0 & 0 & 0 & 0 & 1 & 3 & 3 & 1 & 0 & 0 & 10 \\
2003 & 2 & 0 & 3 & 0 & 0 & 0 & 0 & 0 & 3 & 6 & 1 & 1 & 16 \\
2004 & 1 & 0 & 0 & 0 & 0 & 0 & 0 & 0 & 3 & 3 & 2 & 6 & 15 \\
2005 & 2 & 4 & 0 & 0 & 0 & 0 & 0 & 0 & 0 & 5 & 4 & 1 & 16 \\
2006 & 0 & 2 & 0 & 0 & 0 & 0 & 0 & 0 & 1 & 4 & 5 & 4 & 16 \\
2007 & 1 & 2 & 1 & 0 & 0 & 0 & 1 & 1 & 6 & 18 & 7 & 1 & 38 \\
\hline Total & 10 & 8 & 5 & 1 & 1 & 0 & 3 & 5 & 17 & 43 & 20 & 16 & 129 \\
\hline Média & 1 & 0,8 & 0,5 & 0,1 & 0,1 & 0 & 0,3 & 0,5 & 1,7 & 4,3 & 2,0 & 1,6 & \\
\hline
\end{tabular}

No presente estudo, a verificação destes 129 nascimentos durante os 10 anos de levantamento dos dados foi possível observar uma frequência maior de nascimento no mês de outubro (33\%) (Gráfico 1), semelhantes ao que foi descrito por Guimarães et al. (2008) em Agouti paca na região norte do Brasil, entretanto estudos com a mesma espécies na região sudeste do Brasil mostram resultados diferentes, nos quais observaram que os nascimentos desses animais em cativeiro ocorreram com uma frequência maior nos meses de julho (inverno) e entre novembro e janeiro (verão), sendo que nos meses de fevereiro, março e agosto não ocorreram nascimentos (Guimarães et al., 2008).

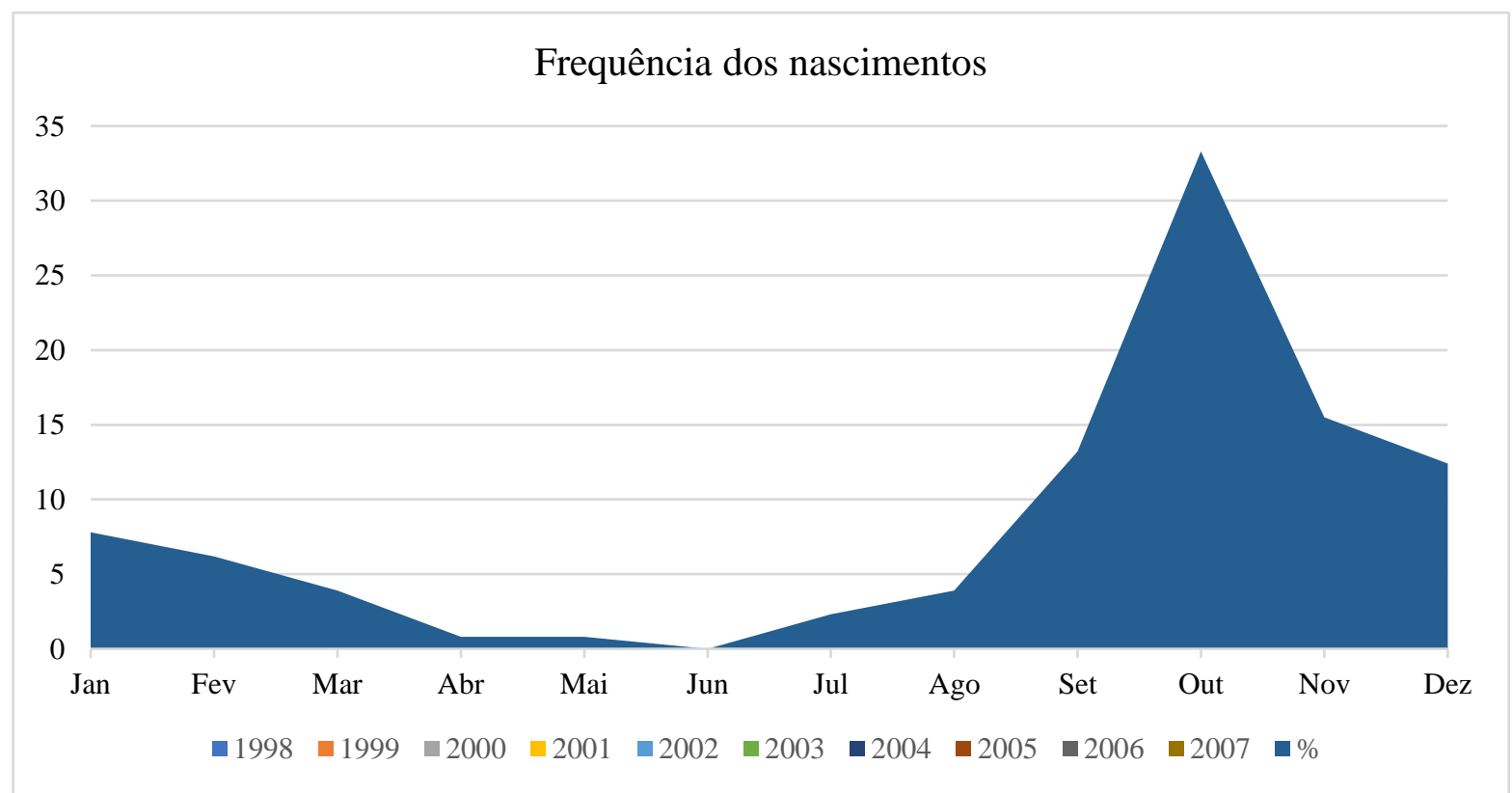

Gráfico 1. Frequência dos nascimentos de cutias (Dasyprocta prymnolopha) nascidas no NEPAS na UFPI-Teresina, no período de 1998 a 2007. 
Foi observado que, com o aumento no interesse na criação de animais silvestres em cativeiro, associado ao aumento de pesquisas nesta área, a eficiência reprodutiva desses animais aumentou ao longo dos anos no ambiente deste estudo. Em 1998, houve o registro de apenas dois partos, enquanto que em 2007 esse número foi para 38 partos no ano (Tabela 1), todavia esses dados também estão correlacionados ao aumento do número de animais no plantel do NEPAS.

Ao observar o intervalo entre partos, foram verificados que, 33 fêmeas tiveram o intervalo entre partos de até um ano, 25 fêmeas tiveram o intervalo entre um a dois anos, seis fêmeas entre dois a três anos, duas fêmeas entre seis a sete anos e 37 fêmeas tiveram apenas um parto durante a sua vida. Podemos inferir que essa diferença de partos entre os animais pode estar relacionada ao comportamento de dominância de fêmeas em relação às outras. No mesmo recinto chegam a inibir o estro ou mesmo impedir o cruzamento de fêmeas submissas com o macho. Assim como, também alguma disfunção reprodutiva, conforme observações feitas por Rêgo et al. (2019) em grupos de cutias (D. prymnolopha) criadas em cativeiros, onde encontraram comportamentos mais agressivos entre as fêmeas em relação ao seu local de descanso e alimentos, como também perseguições de fêmeas dominantes a fêmeas submissas e a machos.

Já em estudos realizados por Carleton \& Musser (2005), os resultados implicam que a frequência e o tipo de parto não sofrem influência da época do ano, classificando as fêmeas Dasyprocta sp. em poliéstricas contínuas. Em seus estudos com Paca (Agouti paca), Nogueira (2005) observou que apenas $40 \%$ das fêmeas pariram duas vezes ao ano e $60 \%$ uma única vez. Guimarães et al. (2008) observaram que, em 10 fêmeas estudadas, 55,6\% tiveram dois partos por ano e 44,4\% apenas um parto, em um intervalo entre partos com média de 224,5 + 52,2 dias (115 - 326 dias) em 18 observações. Em fêmeas mantidas em cativeiro em condições similares, $34,8 \%$ das fêmeas obtiveram um parto, $21,7 \%$ dois partos, 13\% três partos e 30,4\% nenhum, por ano, com variação entre 97 a 101 dias, embora, também tenha relatado intervalos superiores a 178 e 190 dias (Matamoros, 1982).

O tipo de parto em cutias geralmente é o simples, podendo ocorrer o parto duplo e até parto triplo. Neste levantamento foi verificado um total de 149 partos, onde desses, $85 \%$ foram de partos simples (127 fêmeas), $13 \%$ de partos duplos (20 fêmeas) e 1\% de parto triplo (2 fêmeas). Embora sejam variação citadas para o gênero, é visto na literatura percentuais diferentes dos nossos achados. Em estudos, Cavalcante et al. (2006) constaram 48,5\% de partos simples e 51,5\% duplo, Lopes et al. (2004) observaram $29,1 \%$ de parto simples e 70,6\% parto duplo, Oliveira et al. (2001) relatam 61,7\% de partos duplos, Guimarães (1993) com 65,8\% partos duplos, e nos estudos de Hosken \& Silveira (2001) a maioria das vezes os partos foram gemelares.

Os dados dos fatores climáticos e a média dos nascimentos estão representados na Tabela 2, bem como a correlação entre estes dados estão descritos na tabela 3.

A atividade reprodutiva da cutia, assim como em muitos animais, pode estar associada a fatores ambientais, climáticos, reprodutivos e fisiológicos, porém, como visto na tabela 3 ocorreu uma forte correlação às condições ambientais, como temperatura e umidade. Em estudos com espécies tropicais de roedores de vida livre, na Guiana francesa, percebeu-se uma sincronia entre o período chuvoso e a disponibilidade de frutas no ambiente, independentemente do estado corpóreo, necessidades metabólicas e período de gestação das espécies. Indicando que fatores ambientais no momento do nascimento são mais importantes para a reprodução dessas espécies tropicais (Dubost \& Henry, 2017).

A literatura indica que características sazonais refletem na atividade gonadal em machos e fêmeas, muitas vezes de forma distinta, determinando os nascimentos de suas proles em períodos do ano propícios a sobrevivência (Bronson, 1985; Horton, 2005). Algumas espécies usam este sincronismo da reprodução como estratégia para otimizar o seu sucesso (Ims, 1990). De acordo com Horton (2005), as condições ambientais podem imprimir sazonalidade em uma determinada espécie, ou influenciar o animal individualmente, onde a reprodução ocorrerá variavelmente em qualquer período do ano, dependendo dessas condições. Podem, ainda, imprimir características genéticas, passada de geração em geração, onde os animais jovens já apresentam, em suas funções reprodutivas, a idade de tornarem-se púberes e o período propício para reproduzir-se. 
Tabela 2. Valores médios mensais dos fatores climáticos e nascimentos de cutias (D. prymnolopha) criadas no NEPAS entre os anos de 1998 a 2007.

\begin{tabular}{lccccc|c}
\hline Meses & \multicolumn{7}{c}{ Dados climáticos } \\
\cline { 2 - 7 } & Tmáx. & Tmin. & UR & PP & TM & Nasc. \\
\hline Janeiro & 32,68 & 23,17 & 79,5 & 220,1 & 27,94 & 1 \\
Fevereiro & 31,94 & 22,98 & 82,9 & 246,2 & 27,46 & 0,8 \\
Março & 31,85 & 23,09 & 84,36 & 271,1 & 27,49 & 0,5 \\
Abril & 31,79 & 23,1 & 84,48 & 218,9 & 27,49 & 0,1 \\
Maio & 32,26 & 22,92 & 80,34 & 91,36 & 27,62 & 0,1 \\
Junho & 32,47 & 22,05 & 73,62 & 20,71 & 27,25 & 0 \\
Julho & 33,56 & 21,49 & 67,78 & 8,61 & 27,51 & 0,3 \\
Agosto & 33,29 & 21,06 & 59,27 & 11,49 & 28,2 & 0,5 \\
Setembro & 36,49 & 22,52 & 55,59 & 15,24 & 29,52 & 1,7 \\
Outubro & 37,38 & 23,57 & 53,97 & 14,24 & 30,47 & 4,3 \\
Novembro & 36,37 & 23,93 & 59,21 & 60,82 & 30,12 & 2,0 \\
Dezembro & 34,58 & 23,73 & 68,26 & 85,58 & 29,12 & 1,6 \\
\hline
\end{tabular}

TMA-Temperatura máxima; TMI-Temperatura mínima; UR-Umidade relativa do ar; PP-Precipitação pluviométrica; TMTemperatura média; NASC- Nascimento.

Tabela 3. Correlação simples entre as médias dos fatores climáticos e dos nascimentos de cutias criadas em cativeiro no NEPAS entre 1998 a 2007.

\begin{tabular}{lcc}
\hline & COEF.CORR® & SIGNIF. \\
\hline Temperatura máxima x Nascimento & 0,7998 & $* *$ \\
Temperatura mínima x Nascimento & 0,5110 & Ns \\
Umidade relativa do ar x Nascimento & $-0,6690$ & $*$ \\
Precipitação pluviométrica x Nascimento & $-0,3041$ & $\mathrm{Ns}$ \\
Temperatura média x Nascimento & 0,9003 & $* *$ \\
\hline
\end{tabular}

** significativo ao nível de $1 \%$ de probabilidade $(\mathrm{P}<0,10)$; *significativo ao nível de $5 \%$ de probabilidade $(0,01<\mathrm{P}<0,05)$; Ns-não significativo $(\mathrm{P}>0,05)$.

Outro dado relacionado ao aumento do número de nascimentos nos períodos mais quentes do ano em cutias criadas em cativeiro em Teresina-PI no período de 1998 a 2007, pode ser atribuído às observações feitas por Menezes (2017), onde a cutia macho apresenta maior atividade espermatogênica durante o período de menor precipitação de chuvas e temperaturas mais elevadas, com melhor potencial reprodutivo para esta época do ano, fato semelhante ao observado em outros roedores selvagens que vivem em ambientes com características sazonais de acordo com o autor.

\section{Conclusão}

O número de animais nascidos é significativamente maior nos meses de julho a fevereiro, com a maior concentração no mês de outubro. Os dados apontaram que os nascimentos ocorreram em períodos mais quentes do ano e com um volume menor de chuvas, havendo assim, uma correlação positiva entre as temperaturas, a umidade relativa do ar em relação ao aumento nos índices de nascimento, o que podemos sugerir que há uma forte tendência desses animais a reproduzirem-se nessas condições ambientais. Considerando a discrepância de dados na literatura em relação à existência de um período de sazonalidade ou não desses animais, com relação também na frequência dos nascimentos e do tipo de parto, outros trabalhos devem ser desenvolvidos no sentido de corroborar nossos achados, bem como dos fatores envolvidos que podem influenciar o ciclo reprodutivo anual de cutias.

\section{Agradecimentos}

A Universidade Federal de Campina Grande pelo o apoio do programa institucional de Voluntários de Iniciação Científica. 
Aos colegas do Grupo de Pesquisa da Morfologia Veterinária, ao Laboratório de Anatomia Veterinária da Unidade Acadêmica de Medicina Veterinária, onde este trabalho foi desenvolvido, por todo o apoio e contribuição.

A Universidade Federal do Piauí por ceder os dados que embasam esta pesquisa.

A EMBRAPA Meio Norte pelo fornecimento dos dados climáticos.

\section{Referências bibliográficas}

Almeida, M. M., Carvalho, M. A. M., Cavalcante Filho, M. F., Miglino, M. A. \& Menezes, D. J. A. (2003). Estudo morfológico e morfométrico do ovário de cutias (Dasyprocta aguti, Linnaeus, 1766). Brazilian Journal of Veterinary Research and Animal Science, 40(1):55-62.

Bronson, F. H. (1985). Mammalian reproduction: an ecological perspective. Biology of Reproduction, 32(1):1-26.

Carleton, M. D. \& Musser, G. G. (2005). Order Rodentia. In D. E. Wilson, D. M. Reeder, R. R. Cavalcante, M. M. Almeida, S. G. Moura \& L. M. Martins Júnior (Eds.), Mammal Species of the World. A Taxonomic and Geographic Reference (pp. 745-752). Baltimore, USA: JHU Press.

Cavalcante, R. R., Almeida, M. M., Moura, S. G., Júnior, L. M. M., Conde Júnior, A. M., Carvalho, M. A. M. \& Lopes, J. B. (2006). Peso pós-parto, frequência e prevalência do tipo de parto de cutias (Dasyprocta sp.) criadas em cativeiro. Ciência Animal Brasileira, 6(2):67-70.

Dubost, G. \& Henry, O. (2017). Seasonal reproduction in Neotropical rainforest mammals. Zoological Studies, 56(2):1-9.

Guimarães, D. A. A. (1993). Algumas características reprodutivas da cutia fêmea Dasyprocta prymnolopha (Wagler, 1831) criada em cativeiro. Master of Science, Universidade Federal do Pará, Belém.

Guimarães, D. A. A., Bastos, L. V., Ferreira, A. C. S., Luz-Ramos, R. S., Ohashi, O. M. \& Ribeiro, H. L. (2008). Características reprodutivas da paca fêmea (Agouti paca) criada em cativeiro. Acta Amazônica, 38(3):531-538.

Horton, T. H. (2005). Fetal origins of developmental plasticity: Animal models of induced life history variation. American Journal of Human Biology, 17(1):34-43.

Hosken, F. M. \& Silveira, A. C. (2001). Criação de cutias. Viçosa, Minas Gerais, Brasil.: Aprenda Fácil.

Ims, R. A. (1990). The ecology and evolution of reproductive synchrony. Trends in Ecology \& Evolution, 5(5):135-140.

Köppen, W. \& Geiger, R. (1928). Klimate der Erde. Gotha: Verlag Justus Perthes. Wall-map $150 \mathrm{~cm} \times 200 \mathrm{~cm}$.

Lange, R. R., Monteiro Filho, E. L. A., Margarido, T. C. C., Pachaly, J. R. \& Mangif, P. R. (2014). Azame Lichtenstein, 1823 (Rodentia: Mammalia).

Lange, R. R. \& Schmidt, E. M. S. (2014). Rodentia - Roedores Selvagens (Capivara, Cutia, Paca e Ouriço). In Z. S. Cubas, J. C. R. Silva \& J. L. Catão-Dias (Eds.), Tratado de animais selvagens: medicina veterinári. São Paulo, Brasil: Roca.

Lopes, J. B., Cavalcante, R. R., Almeida, M. M., Carvalho, M. A. M., Moura, S. G., Dantas Filho, L. A. \& Conceição, W. L. F. (2004). Desempenho de cutias (Dasyprocta prymnolopha) criadas em cativeiro do nascimento até o desmame em Teresina, Piaú. Revista Brasileira de Zootecnia, 33(6):2318-2322.

Matamoros, Y. (1982). Notas sobre la biologia del Tepezcuintle, Cuniculus paca, Brisson (Rodentia: Dasyproctidae) en cautiveirio. Brenesia, 19/2071-82.

Menezes, D.J.A., Silva, A.R.N., Vieira, F.A.S.Silva Neto, R.B., Oliveira, M.F., Illera, M.J., Assis Neto, A.C., Santos, J.R.S. \& Carvalho, M.A.M (2017). Morfologia e dinâmica testicular em cutias - Dasyprocta prymnolopha) adultas. Arquivo Brasileiro de Medicina Veterinária e Zootecnia, 69(4), 997-1005. 
Meritt Junior, D. A. (1983). Preliminary observations on reproduction in the Central American agouti, Dasyprocta punctata. Zoo Biology, 2(2):127-131.

Nogueira, T. M. R., Toniollo, G. H. \& Giannoni, M. L. (2005) Estrous cycle colpocytology in captive pacas (Agouti paca, Linnaeus, 1766). Ars Veterinaria, 21(4), 209-214.

Oliveira, M. F., Macedo, C. R. \& Feijó, F. M. C. (2001). Dados do manejo reprodutivo de cutias criadas em cativeiro (Dasyprocta aguti). Paper presented at the Congresso da Associação Brasileira de Veterinários de Animais Selvagens, São Paulo, Brasil.

Pinheiro, M. J. P., Andrade, S. A. \& Cunha, J. N. (1989). Preservação e exploração de animais silvestres nativos: preá, cutia e mocó. Revista Caatinga, 6(1):28-49.

Ptaszynska, M. (2008). Compêndio de Reprodução Animal-Intervet. 2010.

Rêgo, J. F., Carvalho, M. A. M., La Salles, A. Y. F. \& Menezes, D. J. A. (2019). Descrição das categorias de comportamentos do Dasyprocta prymnolopha em condições de cativeiro. PUBVET, 13(7):1-9.

Rodrigues, R. F., Miglino, M. A., Ferraz, R. H. S. \& Morais-Pinto, L. (2003). Placentação em cutias (Dasyprocta aguti, CARLETON, MD): aspectos morfológicos. Brazilian Journal of Veterinary Research and Animal Science, 40(2):133-137.

Roth-Kolar, H. (1957). Beiträge zu einem Aktionssystem des Aguti (Dasyprocta aguti aguti L.). Zeitschrift für Tierpsychologie, 14(3):362-375.

Silva, F. A. S. 2016. ASSISTAT: Versão 7.7 beta. DEAG-CTRN-UFCG - Atualizado em 01 de agosto de 2016. Disponível em <http://www.assistat.com/>. Acessado em: 01 de agosto de 2016Weir, B. J. (1971). Some observations on reproduction in the female agouti, Dasyprocta aguti. Reproduction, 24(2):203-211.

Zuckerman, S. (1952). The breeding seasons of mammals in captivity. Paper presented at the Proceedings of the Zoological Society of London.

Recebido: 22 de outubro, 2019.

Aprovado: 26 de novembro, 2019.

Publicado: 23 de janeiro, 2020.

Licenciamento: Este artigo é publicado na modalidade Acesso Aberto sob a licença Creative Commons Atribuição 4.0 (CC-BY 4.0), a qual permite uso irrestrito, distribuição, reprodução em qualquer meio, desde que o autor e a fonte sejam devidamente creditados. 